\title{
GLOBAL SOLUTIONS TO THE LAKE EQUATIONS WITH ISOLATED VORTEX REGIONS
}

BY

\author{
CHAOCHENG HUANG
}

Department of Mathematics, Wright State University, Dayton, Ohio 45435

\begin{abstract}
The vorticity formulation for the lake equations in $R^{2}$ is studied. We assume that the initial vorticity has the form $\omega(x, 0)=\omega_{0}(x) \chi_{\bar{\Omega}_{0}}$, where the initial vortex region $\Omega_{0}$ is a $C^{1+\alpha}$ domain and $\omega_{0} \in C^{\alpha}\left(\bar{\Omega}_{0}\right)$. It is shown that the Cauchy problem can be formulated as an integral system. Global existence and uniqueness of the $C^{1+\alpha}$ solution to the integral system are established. Consequently, the lake equation admits a unique weak solution, global in time, in the form of $\omega(x, t)=\omega_{t}(x) \chi_{\bar{\Omega}_{t}}$, where $\omega_{t}(x) \in C_{x}^{\alpha}\left(\bar{\Omega}_{t}\right)$ and $\partial \Omega_{t} \in C^{\alpha}$.
\end{abstract}

1. Introduction. Consider the circulation of an inviscid fluid in a very large shallow basin with a varying bottom. Let $x=\left(x_{1}, x_{2}\right) \in R^{2}$ be the horizontal position inside the basin and let $t$ be the time; then the evolution of vertically averaged fluid velocity vector $u(x, t)=\left(u_{1}(x, t), u_{2}(x, t)\right) \in R^{2}$ is governed by the great lake equations $[2,11,12]$ :

$$
\begin{aligned}
& v_{t}=u^{\perp} \nabla \wedge v+\nabla\left(h-\frac{1}{2}|u|^{2}+u \cdot v\right), \nabla \cdot(b u)=0, \\
& v=u+\delta^{2}\left((u \cdot \nabla b) \nabla b+\frac{1}{2} b(\nabla \cdot u) \nabla b-\frac{1}{2 b} \nabla\left(b^{2} u \cdot \nabla b\right)-\frac{1}{3 b} \nabla\left(b^{3} \nabla \cdot u\right)\right),
\end{aligned}
$$

where $h(x, t)$ is the surface height variation, $b(x)$ is the depth of the basin, $u^{\perp}=$ $\left(u_{2},-u_{1}\right), \nabla \wedge u=\partial_{1} u_{2}-\partial_{2} u_{1}$, and $\delta$ is the aspect ratio- the ratio of vertical to horizontal length scales. In the 0 th order approximation in $\delta^{2}$, we obtain $v=u$. The great lake equation then reduces to the lake equation

$$
u_{t}=u^{\perp} \nabla \wedge v+\nabla\left(h-\frac{1}{2}|u|^{2}+u \cdot v\right), \nabla \cdot(b u)=0 .
$$

Received February 15, 2000.

2000 Mathematics Subject Classification. Primary 35Q35.

E-mail address: chuang@math.wright.edu 
Introducing the potential vorticity $\omega=b^{-1} \nabla \wedge u$ and applying the curl in the first equation above, we arrive at the following system:

$$
\begin{aligned}
& \omega_{t}(x, t)+u(x, t) \cdot \nabla \omega(x, t)=0, \\
& b(x) \omega(x, t)=\nabla \wedge u(x, t), \\
& \nabla \cdot(b(x) u(x, t))=0 .
\end{aligned}
$$

We refer to [12] for detailed description about the great lake equations and lake equations.

The system (1.1)--(1.3) is closely related to the 2D incompressible Euler equation. In fact, when $b(x) \equiv 1$, this system reduces to the vorticity equation for the $2 \mathrm{D}$ Euler equation. Hence, system (1.1)-(1.3) may be viewed as the vorticity formulation of the lake equation. The lake equation in a bounded domain $\Omega$ has been studied in $[10,11,12,14]$. In those papers, the authors analyzed even more general systems in which (1.2) is replaced by $u=K \omega$ for certain linear operators $K$. They established global existence of solutions in the Sobolev spaces $W^{m, p}$ and in the space of analytical functions. For the studies of the $2 \mathrm{D}$ Euler equation, we refer to [13] and references therein.

We are interested in the situation when the basin is very large with an isolated initial vortex region. This case may be approximated by the vorticity formulation of the lake equation (1.1)-(1.3) in the whole space with the initial vorticity of the form

$$
\omega(x, 0)=\omega_{0}(x) \chi_{\bar{\Omega}_{0}},
$$

where $\chi_{\bar{\Omega}_{0}}$ is the characteristic function of $\bar{\Omega}_{0}$, the closure of a domain $\Omega_{0}$, and $\omega_{0}(x) \geq 0$ is a given function. We assume that the initial vortex region $\Omega_{0}$ is a bounded $C^{1+\alpha}$ domain, and that $\omega_{0}(x)$ is $\alpha$-Hölder continuous in $\bar{\Omega}_{0}$. Note that $\omega_{0}(x)$ may be extended to the whole space. Hence, the last assumption is equivalent to $\omega_{0}(x)$ being $\alpha-$ Hölder continuous in $R^{2}$. We also note that when $\omega_{0}\left(x_{0}\right)>0$ for some $x_{0} \in \partial \Omega_{0}$, the initial vorticity $\omega(x, 0)$ is discontinuous at $x_{0}$. Hence, the initial condition (1.4) includes, in particular, an interesting situation that the initial vorticity may be discontinuous across the boundary $\partial \Omega_{0}$ of $\Omega_{0}$.

For the 2D Euler equation, existence of global weak solutions for general initial data was established in [15]. For the Cauchy problem with initial data (1.4), the problem of global regularity for weak solutions was proposed by Majda in [13]. In particular, when $\omega_{0}(x)$ is a constant, a weak solution is referred to as a constant vortex patch. Global regularity of constant vortex patches was established by Chemin [3]. Roughly speaking, Chemin asserts that the initial regularity of the vortex region for a constant vortex patch will persist for all time. A simpler proof of more general regularity results can be found in [1] and [7].

In this paper, we are concerned with global existence and stratified regularity of weak solutions to (1.1)-(1.4). The main purpose is to extend Chemin's regularity result to the lake equation. We shall present an integral equation approach to the Cauchy problem for the lake equation. In this approach, the vorticity equation will be formulated as a non-local integral system in $\bar{\Omega}_{0} \times[0, \infty)$. We shall show that there exists a unique $C^{1+\alpha}$ solution $\Phi(x, t)$ to the integral system for all $t \geq 0$. This $C^{1+\alpha}$ solution $\Phi(x, t)$ will naturally produce a unique weak solution to the lake equation (1.1)-(1.4) by $\omega(x, t)=\omega_{0}\left(\Phi^{-1}(x, t)\right) \chi_{\tilde{\Omega}_{t}}$, where $\Omega_{t}=\Phi\left(\Omega_{0}, t\right)$ is the vortex region. Clearly, this 
implies immediately that the vortex region is a $C^{1+\alpha}$ domain and the vorticity enjoy the stratified regularity of Hölder types, i.e., $\omega(\cdot, t) \in C^{\alpha}\left(\bar{\Omega}_{t}\right)$, for any fixed $t>0$.

The integral equation method was previously used to study dynamics of charged particles in [5] and superconductor vortices in [8]. The method employed in [1] for 2D constant vortex patches is essentially an integral equation method, though the authors there did not explicitly deal with the integral equation for $\Phi(x, t)$. For local existence, we shall use a fixed point argument as in [5]. However, the integral system for the lake equation is structurally different from those in [5]. Some additional non-trivial technical treatments are necessary in order to establish existence and uniqueness of solutions of (1.1)-(1.4) for a short time. The treatment for global existence and regularity is inspired by an idea in [1] and [7]. As we demonstrated in [5], existence of global solutions for these types of integral systems depends on their structures and initial data. Fortunately, for the lake equation, the singular part of the integral kernel is similar to the one for the Euler system. Though the initial vorticity $\omega_{0}(x)$ is no longer a constant here, we are still able to establish various estimates that show, for some $0<\beta<\alpha$, the logarithmically superlinear growth for the $\beta$-Hölder norms of the vorticity and the boundary of the vortex region. These estimates then lead to á prióri estimates for the $\alpha$-Hölder norms of the above quantities. The results on global existence and regularity follow immediately.

The paper is organized as follows. In the next section, we shall derive the integral formulation for the lake equation (1.1)-(1.4). We shall show that in a certain sense, a solution of the integral equation will produce naturally a weak solution to (1.1)-(1.4). In Sec. 3, we shall study short-time existence, uniqueness, and regularity of solutions to the integral equation. Finally in Sec. 4, we shall establish existence and regularity for all $t>0$.

2. Integral System Formulation. In this section, we shall formulate the system (1.1)-(1.4) as a non-local integral system in the sense that any solution of the integral system defines a unique weak solution. To illustrate this integral formulation, we may assume that $\omega(x, t)$ is a smooth solution of (1.1)-(1.4) with the smooth velocity field $u(x, t)$. This is the case when $\omega_{0}(x)$ in (1.4), defined only in $\bar{\Omega}_{0}$, is smooth and has a compact support in $\Omega_{0}$ (hence $\omega(x, 0)$ is smooth), and when $b(x) \in C_{b}^{\infty}$ (the space of smooth functions with bounded derivatives) and $b(x) \geq b_{0}>0$ for some constants $b_{0}$. Since $\nabla \cdot(b u)=0$, there exists a potential function $\psi(x, t)$ such that

$$
b(x) u(x, t)=\nabla^{\perp} \psi(x, t) \stackrel{\text { def }}{=}\left(\partial_{2} \psi(x, t),-\partial_{1} \psi(x, t)\right) .
$$

By (1.2), this potential satisfies

$$
\nabla \wedge u=\nabla \wedge\left(b^{-1} \nabla^{\perp} \psi\right)=b \omega
$$

or equivalently, the following elliptic equation

$$
-\Delta \psi(x, t)+\nabla \log b(x) \cdot \nabla \psi(x, t)=b(x)^{2} \omega(x, t),
$$

for fixed $t$, with the growth condition at infinity: $\psi(x, t) \rightarrow O(\log |x|)$ as $|x| \rightarrow \infty$. Suppose that the elliptic operator $-\Delta+\nabla \log b \cdot \nabla$ in (2.2) has the fundamental solution, 
say $K(x, y)$. Then

$$
\psi(x, t)=\int_{R^{n}} K(x, z) b^{2}(z) \omega(z, t) d z .
$$

Next, we define the fluid particle trajectory $\Phi(x, t)$, for fixed $x \in R^{2}$, by

$$
\frac{\partial \Phi}{\partial t}=u(\Phi, t), \quad \Phi(x, 0)=x .
$$

It follows from $(1.1)$ that $\omega(\Phi(x, t), t)$, for fixed $x$, solves the equation

$$
\frac{d \omega}{d t}=0
$$

Hence $\omega(\Phi(x, t), t)=\omega(x, 0)$, or $\omega(x, t)=\omega\left(\Phi^{-1}(x, t), 0\right)$. Set

$$
\Omega_{t}=\Phi\left(\Omega_{0}, t\right)=\left\{\Phi(z, t): z \in \Omega_{0}\right\} .
$$

Then, we may write the vorticity in the form

$$
\omega(x, t)=\omega_{0}\left(\Phi^{-1}(x, t)\right) \chi_{\bar{\Omega}_{t}} .
$$

Substituting (2.1), (2.3), and (2.5) into (2.4), we obtain the dynamical system

$$
\begin{aligned}
\frac{\partial \Phi(x, t)}{\partial t} & =\frac{1}{b(\Phi(x, t))} \int_{\Omega_{t}} \nabla_{x}^{\perp} K(\Phi(x, t), z) b(z)^{2} \omega_{0}\left(\Phi^{-1}(z, t)\right) d z, \\
\Phi(x, 0) & =x
\end{aligned}
$$

where $\Phi^{-1}$ is the inverse of the mapping $x \mapsto \Phi(x, t)$ for any fixed $t$. This system is nonlocal in the sense that the value $\Phi\left(x_{0}, t\right)$ at $x_{0}$ depends on $\Phi(x, t)$ for all $x$. This equation can also be written equivalently as the integral system

$$
\Phi(x, t)=x+\int_{0}^{t} \int_{\Omega_{s}} \frac{\nabla_{x}^{\perp} K(\Phi(x, s), z) b(z)^{2} \omega_{0}\left(\Phi^{-1}(z, s)\right)}{b(\Phi(x, s))} d z d s .
$$

This integral system clearly depends only on $\Phi(x, t)$ in $\bar{\Omega}_{0} \times[0, \infty)$. It is easy to see that the above procedure can be reversed. In other words, suppose that $\Phi(x, t)$, defined in $\bar{\Omega}_{0} \times[0, \infty)$, is smooth and it solves (2.7). Define $\omega$ by $(2.5), \psi$ by $(2.3)$, and then $u$ by (2.1). A direct computation shows that $(\omega, u)$ solve (1.1)-(1.4). Hence, for smooth initial data, the integral system (2.7) is equivalent to (1.1)-(1.4).

In general, for the initial vorticity $\omega(x, 0)$ in $(1.4)$ that has jump discontinuity along $\partial \Omega_{0}$, one expects from $(2.5)$ that $\omega(x, t)$ also has jump discontinuity along $\partial \Omega_{t}$. Since the kernel $\nabla_{x}^{\perp} K(x, y)$ in $(2.7)$ is singular as $x \rightarrow y$, the integral on the right-hand side of (2.7) may not be smooth as $x$ crosses $\partial \Omega_{0}$. Hence, we shall look for solutions of $(2.7)$ with less regularity. To this end, we need to introduce some notations.

For any family of open subsets $\left\{G_{t}\right\}_{t>0}$ in $R^{2}$, integer $m \geq 0$, and $0 \leq \alpha<1$, denote by $C_{x}^{m+\alpha}\left(G_{t}\right)$ the set of all functions $f(x, t)$ defined for $x \in G_{t}$ such that $f(\cdot, t)$ and all the spatial derivatives up to $m$ th order are $\alpha$-Hölder continuous in $G_{t}$. Denote by $C_{x}^{m+\alpha}\left(\bar{G}_{t}\right)$ the set of extensions to $\bar{G}_{t}$ of all functions in $C_{x}^{m+\alpha}\left(G_{t}\right)$. Denote by $|f(t)|_{m+\alpha}$ the Hölder semi-norm defined as

$$
|f(t)|_{m+\alpha}=\sup _{x, y \in G_{+} \cdot|\gamma|=m} \frac{\left|\partial_{\gamma} f(x, t)-\partial_{\gamma} f(y, t)\right|}{|x-y|^{\alpha}},
$$


where $\gamma=\left(\gamma_{1}, \gamma_{2}\right)$ is a multi-index, $\partial_{\gamma}=\partial_{x_{1}}^{\gamma_{1}} \partial_{x_{2}}^{\gamma_{2}}$. The Hölder norm is denoted by

$$
\|f(t)\|_{m+\alpha}=\sup _{x \in G_{t},|\gamma| \leq m}\left|\partial_{\gamma} f(x, t)\right|+|f(t)|_{m+\alpha} .
$$

Sometime we shall use the notations $|f(t)|_{m+\alpha, G_{t}}$ and $\|f(t)\|_{m+\alpha, G_{t}}$ to specify the dependence on the domain $G_{t}$. For convenience, we introduce the notation

$$
|f(t)|_{\text {inf }, \partial G_{t}}=\inf _{x \in \partial G_{t}}|f(x, t)| .
$$

We shall also adopt the notation $W_{l o c}^{m, p}$ for the local Sobolev spaces.

Definition 2.1. We call $\Phi(x, t)$ a $C^{1+\alpha}$ solution of $(2.7)$ in $\bar{\Omega}_{0} \times[0, T)$ if $\Phi, \partial_{t} \Phi \in$ $C_{x}^{1+\alpha}\left(\bar{\Omega}_{0}\right)$, and $\Phi^{-1} \in C_{x}^{1}\left(\bar{\Omega}_{t}\right)$ for $t<T$, and if $(2.7)$ holds everywhere in $\bar{\Omega}_{0} \times[0, T)$.

There are several ways to define weak solutions to (1.1)-(1.4). We shall adopt the following natural definition.

Definition 2.2. A weak solution to (1.1)-(1.4), for $t<T$, is defined as a couple $(\omega, u) \in L^{2} \times W_{l o c}^{1,2}$ such that (1.2) and (1.3) hold in the sense of distributions, and

$$
\begin{aligned}
& \int_{0}^{T} \int_{R^{2}} b(x) \omega(x, t) u(x, t) \cdot \nabla\left(b^{-1} \xi(x, t)\right) d x d t \\
& =-\int_{0}^{T} \int_{R^{2}} \omega(x, t) \xi_{t}(x, t) d x d t-\int_{\Omega_{0}} \omega_{0}(x) \xi(x, 0) d x
\end{aligned}
$$

for any test function $\xi(x, t)$ that has a compact support in $R^{2} \times[0, T)$.

Proposition 2.1. Suppose that $\omega_{0}(x)$ is bounded, $b(x) \in C^{1}$ and $b_{0} \leq b(x) \leq b_{1}$ for some positive constants $b_{0}$ and $b_{1}$. Let $\Phi(x, t)$ be a $C^{1+\alpha}$ solution of $(2.7)$ in $\bar{\Omega}_{0} \times[0, T)$. Define $\omega(x, t)$ by $(2.5), \psi$ by $(2.3)$, and $u$ by $(2.1)$. Then $(\omega, u)$ is a weak solution of the Cauchy problem for the lake equation (1.1)-(1.4).

Proof. Since $\omega_{0}$ is bounded, we know from $(2.5)$ that $\omega(x, t)$ is bounded. We also know that $\Omega_{t}=\Phi\left(\Omega_{0}, t\right)$ is a domain that is bounded uniformly in $t \leq T_{1}$ for any $T_{1}<T$. By the regularity theory for the elliptic equation (2.2), we have $\psi \in W_{\text {loc }}^{2, p}$ for any $p>1$, and consequently $u \in W_{l o c}^{1, p}$. Obviously, the relations (1.2) and (1.3) hold in the sense of distributions. It suffices to show (2.8) with $T$ being replaced by $T_{1}$. By (2.3) and (2.1), we may write $(2.7)$ as

$$
\Phi(x, t)=x+\int_{0}^{t} u(\Phi(x, s), s) d s .
$$

It follows that $\Phi(x, t)$ solve $(2.4)$, i.e.,

$$
\frac{d \Phi(x, t)}{d t}=u(\Phi(x, t), t)
$$

Denote by $J(\Phi(x, t))$ the Jacobian of the mapping $\Phi(\cdot, t)$. By $(2.5), \omega(\Phi(x, t), t)=$ $\omega_{0}(x) \chi_{\bar{\Omega}_{0}}$. Multiplying this equation by

$$
J(\Phi(x, t)) \frac{d \xi(\Phi(x, t), t)}{d t}
$$


where $\xi$ is a test function such that $\xi\left(x, T_{1}\right)=0$, and then integrating over $\Omega_{0} \times\left(0, T_{1}\right)$, we obtain

$$
\begin{aligned}
& \int_{0}^{T_{1}} \int_{\Omega_{0}} \omega(\Phi(x, t), t) J(\Phi(x, t)) \frac{d \xi(\Phi(x, t), t)}{d t} d x d t \\
& =\int_{0}^{T_{1}} \int_{\Omega_{0}} \omega_{0}(x) J(\Phi(x, t)) \frac{d \xi(\Phi(x, t), t)}{d t} d x d t \\
& =-\int_{0}^{T_{1}} \int_{\Omega_{0}} \omega_{0}(x) \frac{d J(\Phi(x, t))}{d t} \xi(\Phi(x, t), t) d x d t-\int_{\Omega_{0}} \omega_{0}(x) \xi(x, 0) d x,
\end{aligned}
$$

where we have performed integration by parts and have used the fact that $\Phi(x, 0)=x$. By (2.4) and (1.3), we have (see [4, p. 25])

$$
\begin{aligned}
\frac{d J(\Phi(x, t))}{d t} & =(\nabla \cdot u)(\Phi(x, t), t) J(\Phi(x, t)) \\
& =b^{-1}(\nabla \cdot(b u)-(\nabla b) \cdot u)(\Phi(x, t), t) J(\Phi(x, t)) \\
& =-\left(b^{-1}(\nabla b) \cdot u\right)(\Phi(x, t), t) J(\Phi(x, t)) .
\end{aligned}
$$

It follows that

$$
\begin{aligned}
& \int_{0}^{T_{1}} \int_{\Omega_{\Omega_{0}}} \omega(\Phi(x, t), t) J(\Phi(x, t)) \frac{d \xi(\Phi(x, t), t)}{d t} d x d t \\
& =\int_{0}^{T_{1}} \int_{\Omega_{0}} \omega_{0}(x)\left(b^{-1} \nabla b \cdot u\right) J(\Phi(x, t)) \xi(\Phi(x, t), t) d x d t \\
& -\int_{\Omega_{0}} \omega_{0}(x) \xi(x, 0) d x \\
& =\int_{0}^{T_{1}} \int_{\Omega_{t}} \omega(x, t)\left(b^{-1} \nabla b \cdot u\right) \xi(x, t) d x d t-\int_{\Omega_{0}} \omega_{0}(x) \xi(x, 0) d x .
\end{aligned}
$$

In deriving the last equality, we have changed variables $y=\Phi(x, t)$. On the other hand, the left-hand side of (2.9) can be expressed as, after performing the same changes of variables,

$$
\begin{aligned}
& \int_{0}^{T_{1}} \int_{\Omega_{0}} \omega(\Phi(x, t), t) \frac{d \xi(\Phi(x, t), t)}{d t} J(\Phi(x, t)) d x d t \\
& =\int_{0}^{T_{1}} \int_{\Omega_{0}} \omega(\Phi(x, t), t) \xi_{t}(\Phi(x, t), t) J(\Phi(x, t)) d x d t \\
& +\int_{0}^{T_{1}} \int_{\Omega_{0}} \omega(\Phi(x, t), t)(\nabla \xi)(\Phi(x, t), t) \cdot \frac{d \Phi(x, t)}{d t} J(\Phi(x, t)) d x d t \\
& =\int_{0}^{T_{1}} \int_{\Omega_{0}}\left(\omega \xi_{t}(\Phi(x, t), t)+(\omega u \cdot \nabla \xi)(\Phi(x, t), t)\right) J(\Phi(x, t)) d x d t \\
& =\int_{0}^{T_{1}} \int_{\Omega_{t}} \omega(x, t)\left(\xi_{t}(x, t)+(u \cdot \nabla \xi)(x, t)\right) d x d t .
\end{aligned}
$$


From this and (2.9), we obtain

$$
\begin{aligned}
& \int_{0}^{T_{1}} \int_{0}^{T} \int_{\Omega_{t}} \omega(x, t)\left(\xi_{t}(x, t)+(u \cdot \nabla \xi)(x, t)\right) d x d t \\
& =-\int_{\Omega_{0}} \omega_{0}(x) \xi(x, 0) d x+\int_{0}^{T_{1}} \int_{\Omega_{t}} \omega(x, t)\left(b^{-1} \nabla b \cdot u\right)(x, t) \xi(x, t) d x d t .
\end{aligned}
$$

This is the same as $(2.8)$. Hence, the couple $(\omega, u)$ is a weak solution to the lake equation.

3. Short-time Solution for Integral System. Throughout the paper, we assume that $b_{0} \leq b(x) \leq b_{1}$, for some positive constants $b_{0}, b_{1}$, that $b(x) \in C^{1+\alpha}\left(R^{2}\right)$, and that the elliptic operator $-\Delta+\nabla \log b \cdot \nabla$ in (2.2) has the fundamental solution $K(x, y)$ of the form

$$
K(x, y)=\hat{b} \log |x-y|+K_{1}(x, y),
$$

where $\hat{b}$ is a positive constant depending only on $b(x)$, and $K_{1}(x, y)$ is a positive $C_{b}^{3}$ function satisfying

$$
|x-y|\left|K_{1}(x, y)\right|+\left|\nabla_{x}^{2} K_{1}(x, y)\right|+\left|\nabla_{x}^{3} K_{1}(x, y)\right| \leq c_{0}
$$

where and throughout the paper the symbol $c_{0}$ is reserved for constants depending only on the given initial data. However, it may vary in different places. Existence of such fundamental solutions is discussed in detail in [9]. In particular, when $b(x)=b(|x|)$ is radially symmetric, the fundamental solution $K(x, y)=K(|x-y|)$ can be found simply by solving the ordinary differential equation $K^{\prime}(r)=b_{0} b(r) r^{-1}$, where $b_{0}$ is a constant. We also assume that $\omega_{0} \in C^{\alpha}\left(\bar{\Omega}_{0}\right)$ for some $0<\alpha<1$, and that $\Omega_{0}$ is a bounded $C^{1+\alpha}$ domain. For simplicity, we assume that there exists $\varphi_{0} \in C^{1+\alpha}$ such that $\Omega_{0}=\left\{x \in R^{2}: \varphi_{0}(x)<0\right\}$ and $\nabla \varphi_{0} \neq 0$ on $\partial \Omega_{0}$.

Let $\Omega$ be a $C^{1+\alpha}$ domain such that $\Omega=\{x: \varphi(x)<0\}$ for some $C^{1+\alpha}$ functions $\varphi(x)$ with $\nabla \varphi(x) \neq 0$ for $x \in \partial \Omega$. We define

$$
\delta_{\Omega}=\frac{|\nabla \varphi|_{\alpha, \Omega}}{|\nabla \varphi|_{\text {inf }, \partial \Omega}} .
$$

This quantity $\delta_{\Omega}$ may be understood as a $C^{1+\alpha}$ character of the $C^{1+\alpha}$ domain. Sometimes we shall use $\delta_{\Omega, \alpha}=\delta_{\Omega}$ to emphasize the dependence on $\alpha$. Consider the Newtonian potential

$$
V(x)=P_{v} \int_{\Omega} \frac{\sigma(x-y)}{|x-y|^{2}} d y,
$$

where $P_{v}$ means the principal value of the following singular integral,

$$
\frac{\sigma(x)}{|x|^{2}}=\nabla^{2} \log |x|=\frac{1}{|x|^{4}}\left(\begin{array}{cc}
x_{2}^{2}-x_{1}^{2} & -2 x_{1} x_{2} \\
-2 x_{1} x_{2} & x_{1}^{2}-x_{2}^{2}
\end{array}\right),
$$

and

$$
G(x)=\int_{\Omega} \frac{\sigma(x-y)}{|x-y|^{2}}(g(x)-g(y)) d y .
$$


It is well-known that the principal value of the integral in defining $V(x)$ exists. We need the following lemma.

Lemma 3.1. Suppose that $\partial \Omega \in C^{1+\alpha}$ and $g \in C^{\alpha}(\bar{\Omega})$. Then

$$
\begin{aligned}
|V|_{0, \Omega} & \leq c \log \left(2+\delta_{\Omega} d(\Omega)\right) \\
|V|_{\alpha, \Omega} & \leq c \delta_{\Omega} \log \left(2+\delta_{\Omega} d(\Omega)\right) \\
|G|_{\alpha, \Omega} & \leq c|g|_{\alpha, \Omega} \log \left(2+\delta_{\Omega} d(\Omega)\right), \\
|G|_{0, \Omega} & \leq c|g|_{0, \Omega} \log \left(2+\alpha^{-1} d(\Omega)|g|_{\alpha, \Omega}\right),
\end{aligned}
$$

where $d(\Omega)$ is the diameter of $\Omega$, and $c$ is a constant independent of $\Omega, \alpha$, and $g$.

Proof. The proof of (3.4)-(3.6) can be found in [5, Lemma 3.1]. An estimate similar to (3.7) can also be found there. However, the dependence on $\alpha$ is not explicitly given in [5, Lemma 3.1]. Since this dependence plays an essential role in establishing global regularity in the next section, we need to show (3.7). Let $\varepsilon>0$ and $B_{\varepsilon}(x)$ be the ball centered at $x$ with radius $\varepsilon$. We write $G(x)=k_{1}(x)+k_{2}(x)$, where

$$
\begin{aligned}
& k_{1}(x)=\int_{\Omega \backslash B_{\varepsilon}(x)} \frac{\sigma(x-y)}{|x-y|^{2}}(g(x)-g(y)) d y, \\
& k_{2}(x)=\int_{B_{\varepsilon}(x)} \frac{\sigma(x-y)}{|x-y|^{2}}(g(x)-g(y)) d y .
\end{aligned}
$$

Since $|\sigma(x)| \leq c$, where $c$ is a constant independent of $\Omega, \alpha$, and $g$, we have

$$
\begin{aligned}
\left|k_{1}(x)\right| & =c \int_{\Omega \backslash B_{\varepsilon}(x)} \frac{|g(x)-g(y)|}{|x-y|^{2}} d y \\
& \leq c|g|_{0, \Omega} \int_{\varepsilon}^{d(\Omega)} \frac{1}{r} d r=c|g|_{0, \Omega} \log \frac{d(\Omega)}{\varepsilon} \\
\left|k_{2}(x)\right| & =c \int_{B_{\varepsilon}(x)} \frac{|g(x)-g(y)|}{|x-y|^{2}} d y \\
& \leq c|g|_{\alpha, \Omega} \int_{0}^{\varepsilon} \frac{1}{r^{1-\alpha}} d r=c \alpha^{-1}|g|_{\alpha, \Omega} \varepsilon^{\alpha} .
\end{aligned}
$$

The estimate (3.7) follows directly by choosing $\varepsilon^{\alpha}=\alpha /|g|_{\alpha, \Omega}$.

Lemma 3.2. Suppose that $\partial \Omega \in C^{1+\alpha}, \omega(x) \in C^{\alpha}(\bar{\Omega})$, and that $\psi$ is the potential associated with $\omega$ through (2.3), i.e.,

$$
\psi(x, t)=\int_{R^{n}} K(x, z) b^{2}(z) \omega(z) d z .
$$

Then

$$
|\nabla \psi|_{0, \Omega} \leq c\|\omega\|_{0, \Omega} d(\Omega),
$$




$$
\begin{gathered}
\left|\nabla^{2} \psi\right|_{0, \Omega} \leq c\|\omega\|_{0, \Omega} \log \left(2+\delta_{\Omega} d(\Omega)+\alpha^{-1}\|\omega\|_{\alpha, \Omega} d(\Omega)\right), \\
\left|\nabla^{2} \psi\right|_{\alpha, \Omega} \leq c\left(\|\omega\|_{\alpha, \Omega}+\|\omega\|_{0, \Omega} \delta_{\Omega}\right) \log \left(2+\delta_{\Omega} d(\Omega)\right),
\end{gathered}
$$

where $d(\Omega)$ is the diameter of $\Omega$, and $c$ is a constant independent of $\Omega, \alpha$, and $\omega$.

Proof. According to (3.1), we write

$$
\psi(x)=\int_{\Omega} K(x, z) b(z)^{2} \omega(z) d z=\psi_{0}(x)+\psi_{1}(x),
$$

where

$$
\begin{aligned}
& \psi_{0}(x)=\hat{b} \int_{\Omega} \log |x-z| b(z)^{2} \omega(z) d z \\
& \psi_{1}(x)=\int_{\Omega} K_{1}(x, z) b(z)^{2} \omega(z) d z .
\end{aligned}
$$

Since $\left|\nabla K_{1}(x, y)\right| \leq c_{0}|x-y|^{-1}$, we have

$$
\begin{aligned}
|\nabla \psi(x)| & =\int_{\Omega}\left|\nabla K(x, z) b(z)^{2} \omega(z)\right| d z \\
& \leq c\|\omega\|_{0, \Omega} \int_{\Omega}|x-z|^{-1} d z \\
& \leq c\|\omega\|_{0, \Omega} \int_{0}^{d(\Omega)} d \rho=c\|\omega\|_{0, \Omega} d(\Omega) .
\end{aligned}
$$

This is the assertion (3.8). To establish the rest of the estimates (3.9) and (3.10), we note that the second derivatives for the Newtonian potential $\psi_{0}$ have the expression

$$
\begin{aligned}
\nabla^{2} \psi_{0}(x) & =\hat{b} \int_{\Omega} \frac{\sigma(x-z)}{|x-z|^{2}}\left(b(z)^{2} \omega(z)-b(x)^{2} \omega(x)\right) d z \\
& +\pi \hat{b} b^{2}(x) \omega(x) I_{2}+\hat{b} b(x)^{2} \omega(x) V(x),
\end{aligned}
$$

where the function $V(x)$ is defined in Lemma 3.1 and $I_{2}$ is the identity matrix in $R^{2}$. The estimates (3.9) and (3.10) follow directly from Lemma 3.1 and (3.2).

Theorem 3.1. There exists a unique $C^{1+\alpha}$ solution $\Phi(x, t)$ of $(2.7)$ for $0<t<T$, for some $T>0$. Consequently, if we define $\Omega_{t}=\Phi\left(\Omega_{0}, t\right), \omega(x, t)=\omega_{0}\left(\Phi^{-1}(x, t)\right) \chi_{\bar{\Omega}_{t}}, \psi$ by (2.3) and $u$ by $(2.1)$. Then $(\omega, u)$ is the weak solution to the lake equation. Furthermore, $\omega(\cdot, t) \in C^{\alpha}\left(\bar{\Omega}_{t}\right), \Omega_{t} \in C^{1+\alpha}$, for $t<T$.

Proof. For any $M, T>0$ to be chosen later on, define a set $B(M, T)$ of $R^{2}$ value functions in $\bar{\Omega}_{0} \times[0, T)$ as follows:

$$
\begin{aligned}
& B(M, T)=\left\{\Phi(x, t) \in R^{2}:\|\Phi(t)\|_{1+\alpha, \Omega_{0}} \leq M,\right. \\
&\left.\|\Phi(x, \cdot)\|_{\alpha} \leq M,\left|\nabla \Phi(x, t)-I_{2}\right| \leq 1 / 2, \Phi(x, 0)=x\right\} .
\end{aligned}
$$

We then define a mapping $F$ from $B(M, T)$ to a functional space by

$$
F(\Phi)(x, t)=x+\int_{0}^{t} u(\Phi(x, s), s) d s
$$


where

$$
u(x, s)=\frac{1}{b(x)} \int_{\Omega_{s}} \nabla_{x}^{\perp} K(x, y) \omega_{0}\left(\Phi^{-1}(y, s)\right) d y,
$$

$\Omega_{s}=\Phi\left(\Omega_{0}, s\right), K(x, y)$ is the fundamental solution in (3.1). By (3.1), we may decompose $u$ as

$$
u(x, s)=u_{0}(x, s)+u_{1}(x, s)
$$

where

$$
\begin{aligned}
& u_{0}(x, s)=\frac{\hat{b}}{b(x)} \int_{\Omega_{s}} \nabla_{x}^{\perp} \log |x-y| \omega_{0}\left(\Phi^{-1}(y, s)\right) d y, \\
& u_{1}(x, s)=\frac{1}{b(x)} \int_{\Omega_{s}} \nabla_{x}^{\perp} K_{1}(x, y) \omega_{0}\left(\Phi^{-1}(y, s)\right) d y,
\end{aligned}
$$

and $K_{1}$ satisfies $(3.2)$. Since $\left|\nabla \Phi-I_{2}\right| \leq 1 / 2, \Phi^{-1}(\cdot, t)$ exists and maps $\bar{\Omega}_{t}$ onto $\bar{\Omega}_{0}$. The mapping $F$ thus is well defined. The rest of the proof is divided into four steps.

Step 1: The mapping $F$ maps $B(M, T)$ into itself for some $M, T$.

For any $\Phi \in B(M, T)$, by Lemma 3.2 , since $\omega_{0}$ is bounded and $\omega_{0}\left(\Phi^{-1}(y, t)\right) \in$ $C^{\alpha}\left(\bar{\Omega}_{t}\right)$, we have $u(\cdot, t) \in C^{1+\alpha}\left(\bar{\Omega}_{t}\right)$, and

$$
\begin{aligned}
& |\nabla u(t)|_{0, \Omega_{t}} \leq c_{0} \log \left(2+\delta_{\Omega_{t}} d\left(\Omega_{t}\right)+\alpha^{-1}\left\|\omega_{0} \circ \Phi^{-1}(t)\right\|_{\alpha, \Omega_{t}} d\left(\Omega_{t}\right)\right), \\
& |\nabla u(t)|_{\alpha, \Omega_{t}} \leq c_{0}\left(\left\|\omega_{0} \circ \Phi^{-1}\right\|_{\alpha, \Omega_{t}}+\delta_{\Omega_{t}}\right) \log \left(2+\delta_{\Omega_{t}} d\left(\Omega_{t}\right)\right) .
\end{aligned}
$$

Let $\varphi_{0}$ be such that $\Omega_{0}=\left\{\varphi_{0}<0\right\}$. Set $\varphi(x, t)=\varphi_{0}\left(\Phi^{-1}(x, t)\right)$. Then $\Omega_{t}=\{\varphi(x, t)<0\}$. From the definition (3.3), it is easy to see that, for $\Phi \in B(M, T)$, we have

$$
\delta_{\Omega_{t}} \leq c_{0} M^{4+\alpha}, d\left(\Omega_{t}\right) \leq 2|\Phi(t)|_{0} \leq 2 M
$$

and

$$
\left\|\omega_{0}\left(\Phi^{-1}(t)\right)\right\|_{\alpha, \Omega_{t}} \leq\left\|\omega_{0}\right\|_{\alpha, \Omega_{0}}\left\|\Phi^{-1}\right\|_{1, \Omega_{t}}^{\alpha} \leq c_{0} M^{2 \alpha}
$$

where $c_{0}$ is a constant independent of $\Phi, M$, and $T$. It follows from (3.18) and (3.19) that

$$
\begin{aligned}
\|\nabla u(\Phi(\cdot, t), t)\|_{\alpha, \Omega_{0}} & \leq|\nabla u(t)|_{0, \Omega_{t}}+|\nabla u(t)|_{\alpha, \Omega_{t}}|\Phi(t)|_{1, \Omega_{0}}^{\alpha} \\
& \leq c_{0} M^{4+2 \alpha} \log (2+M) .
\end{aligned}
$$

By (3.13), the gradient $\nabla F(\Phi)$ can be expressed as

$$
\nabla F(\Phi)(x, t)=I_{2}+\int_{0}^{t} \nabla u(\Phi(x, s), s) \nabla \Phi(x, s) d s .
$$

Hence, for $t<T$,

$$
\|F(\Phi)(t)\|_{1+\alpha, \Omega_{0}} \leq c_{0}+c_{0} T M^{5+2 \alpha} \log (2+M) .
$$

Analogously we can derive, for fixed $x \in \bar{\Omega}_{0}$,

$$
\|F(\Phi)(x, \cdot)\|_{\alpha} \leq c_{0} T^{1-\alpha} M^{3+\alpha}
$$

and

$$
\left|\nabla F(\Phi)(x, t)-I_{2}\right| \leq c_{0} T M^{2} \log (2+M) .
$$


It is now easy to see that if we choose $M=1+2 c_{0}, T=\left(M^{5+2 \alpha} \log (2+M)\right)^{-1}$, then $F(\Phi) \in B(M, T)$ for any $\Phi \in B(M, T)$. We have just proved that the mapping $F$ maps $B(M, T)$ into itself.

Let $M$ and $T$ be fixed so that $F$ maps $B(M, T)$ into itself, and let $\Phi$ and $\Phi^{*}$ be any two functions in $B(M, T)$. In the rest of the proof, $c_{0}$ is a constant depending only on the initial data and the chosen $M, T$.

Step 2: Estimates of $\left|\left(F(\Phi)-F\left(\Phi^{*}\right)\right)(t)\right|_{0, \Omega_{0}}$.

Set

$$
\rho(x, t)=\Phi(x, t)-\Phi^{*}(x, t)
$$

By (3.13), we have

$$
F(\Phi)(x, t)-F\left(\Phi^{*}\right)(x, t)=\int_{0}^{t} u(\Phi(x, s), s) d s-\int_{0}^{t} u^{*}\left(\Phi^{*}(x, s), s\right) d s,
$$

where $u$ is defined in (3.14) and $u^{*}$ is defined accordingly. By changes of variables $z=\Phi^{-1}(y, s)$ and $z=\Phi^{*-1}(y, s)$ in (3.14) for $u$ and $u^{*}$, respectively, we have

$$
\begin{aligned}
u(x, s) & =\frac{1}{b(x)} \int_{\Omega_{0}} \nabla^{\perp} K(x, \Phi(z, s)) b(\Phi(z, s))^{2} \omega_{0}(z) J(\Phi(z, s)) d z, \\
u^{*}(x, s) & =\frac{1}{b(x)} \int_{\Omega_{0}} \nabla^{\perp} K\left(x, \Phi^{*}(z, s)\right) b\left(\Phi^{*}(z, s)\right)^{2} \omega_{0}(z) J\left(\Phi^{*}(z, s)\right) d z,
\end{aligned}
$$

where $J(\Phi(z, s))$ is the Jacobian of the mapping $\Phi(\cdot, s)$. It follows that

$$
\left|u(\Phi(x, s), s)-u^{*}\left(\Phi^{*}(x, s), s\right)\right| \leq\|\rho(s)\|_{1, \Omega_{0}}+|m(x, s)|
$$

where

$$
m(x, s)=c_{0} \int_{\Omega_{0}}\left|\nabla^{\perp} K(\Phi(x, s), \Phi(z, s))-\nabla^{\perp} K\left(\Phi^{*}(x, s), \Phi^{*}(z, s)\right)\right| d z .
$$

We write $m=m_{1}+m_{2}$, where, for small $\varepsilon>0$ to be chosen later,

$$
\begin{aligned}
& m_{1}=c_{0} \int_{\Omega_{0} \backslash B_{\varepsilon}(x)}\left|\nabla^{\perp} K(\Phi(x, s), \Phi(z, s))-\nabla^{\perp} K\left(\Phi^{*}(x, s), \Phi^{*}(z, s)\right)\right| d z, \\
& m_{2}=c_{0} \int_{B_{\varepsilon}(x)}\left|\nabla^{\perp} K(\Phi(x, s), \Phi(z, s))-\nabla^{\perp} K\left(\Phi^{*}(x, s), \Phi^{*}(z, s)\right)\right| d z .
\end{aligned}
$$

By (3.2) and the fact that $\nabla \Phi^{-1}(x, s)$ and $\nabla \Phi^{*-1}(x, s)$ are bounded, we have

$$
\left|m_{1}(x, s)\right| \leq c_{0} \int_{\Omega_{0} \backslash B_{\varepsilon}} \frac{\|\rho(s)\|_{1, \Omega_{0}}}{|x-z|^{2}} d z d s \leq c_{0}\|\rho(s)\|_{1, \Omega_{0}}(1+|\log \varepsilon|)
$$

and

$$
\left|m_{2}(x, s)\right| \leq c_{0} \int_{B_{\varepsilon}}|x-y|^{-1} d z \leq c_{0} \varepsilon
$$


By taking $\varepsilon=\min \left(1,\|\rho(s)\|_{1, \Omega_{0}}\right)$, we obtain

$$
\begin{aligned}
|m(x, s)| & \leq\left|m_{1}(x, s)\right|+\left|m_{2}(x, s)\right| \\
& \leq c_{0}\|\rho(s)\|_{1, \Omega_{0}}\left(1+\left|\log \|\rho(s)\|_{1, \Omega_{0}}\right|\right) d s .
\end{aligned}
$$

Inserting this into (3.21), it follows that

$$
\begin{aligned}
& \left|u(\Phi(x, s), s)-u^{*}\left(\Phi^{*}(x, s), s\right)\right| \\
& \leq c_{0}\|\rho(s)\|_{1, \Omega_{0}}\left(1+\left|\log \|\rho(s)\|_{1, \Omega_{0}}\right|\right) .
\end{aligned}
$$

Therefore,

$$
\begin{aligned}
& \left|F(\Phi)(x, t)-F\left(\Phi^{*}\right)(x, t)\right| \\
& \leq \int_{0}^{t}\left|u(\Phi(x, s), s)-u^{*}\left(\Phi^{*}(x, s), s\right)\right| d s \\
& \leq c_{0} \int_{0}^{t}\|\rho(s)\|_{1, \Omega_{0}}\left(1+\left|\log \|\rho(s)\|_{1, \Omega_{0}}\right|\right) d s .
\end{aligned}
$$

Step 3: Estimates of $\left|\left(F(\Phi)-F\left(\Phi^{*}\right)\right)(t)\right|_{1, \Omega_{0}}$.

From (3.16) and (3.12), we have

$$
\begin{aligned}
\nabla u_{0}(x, s) & =\hat{b} \nabla \int_{\Omega_{s}} \nabla^{\perp} \log |x-y| \omega(y, s) d y \\
& =\hat{b} \int_{\Omega_{s}} \frac{\sigma^{\perp}(x-y)}{|x-y|^{2}}(\omega(y, s)-\omega(x, s)) d y \\
& +\pi \hat{b} \omega(x, s) I_{2}^{\perp}+\hat{b} \int_{\Omega_{s}} \frac{\sigma^{\perp}(x-y)}{|x-y|^{2}} d y \omega(x, s),
\end{aligned}
$$

where

and

$$
\frac{\sigma^{\perp}(x)}{|x|^{2}}=\nabla \nabla^{\perp} \log |x|=\frac{1}{|x|^{4}}\left(\begin{array}{ll}
-2 x_{1} x_{2} & x_{1}^{2}-x_{2}^{2} \\
x_{2}^{2}-x_{1}^{2} & 2 x_{1} x_{2}
\end{array}\right),
$$

$$
\nabla u_{1}(x, s)=\int_{\Omega_{s}} \nabla \nabla^{\perp} K_{1}(x, y) \omega(y, s) d y .
$$

Accordingly, $u^{*}=u_{0}^{*}+u_{1}^{*}$ and the derivatives have the analogous expressions. Using (3.2) and the same techniques as used in deriving (3.22), it follows that

$$
\left|\nabla u_{1}(\Phi(x, s), s)-\nabla u_{1}^{*}\left(\Phi^{*}(x, s), s\right)\right| \leq c_{0}\|\rho(s)\|_{1, \Omega_{0}} .
$$

By changes of variables, we have

$$
\begin{aligned}
& \int_{\Omega_{s}} \frac{\sigma^{\perp}(\Phi(x, s)-y)}{|\Phi(x, s)-y|^{2}}(\omega(y, s)-\omega(\Phi(x, s), s)) d y \\
& =\int_{\Omega_{0}} \frac{\sigma^{\perp}(\Phi(x, s)-\Phi(y, s))}{|\Phi(x, s)-\Phi(y, s)|^{2}}\left(\omega_{0}(y)-\omega_{0}(x)\right) J(\Phi(y, s)) d y
\end{aligned}
$$


and

$$
\begin{aligned}
& \int_{\Omega_{s}^{*}} \frac{\sigma^{\perp}\left(\Phi^{*}(x, s)-y\right)}{\left|\Phi^{*}(x, s)-y\right|^{2}}\left(\omega^{*}(y, s)-\omega^{*}\left(\Phi^{*}(x, s), s\right)\right) d y \\
& =\int_{\Omega_{0}} \frac{\sigma^{\perp}\left(\Phi^{*}(x, s)-\Phi^{*}(y, s)\right)}{\left|\Phi^{*}(x, s)-\Phi^{*}(y, s)\right|^{2}}\left(\omega_{0}(y)-\omega_{0}(x)\right) J\left(\Phi^{*}(y, s)\right) d y .
\end{aligned}
$$

Note that $\left|J(\Phi(y, s))-J\left(\Phi^{*}(y, s)\right)\right| \leq c_{0}\|\rho(s)\|_{1, \Omega_{0}}$, and that

$$
\begin{aligned}
& \left|\frac{\sigma^{\perp}(\Phi(x, s)-\Phi(y, s))}{|\Phi(x, s)-\Phi(y, s)|^{2}}-\frac{\sigma^{\perp}\left(\Phi^{*}(x, s)-\Phi^{*}(y, s)\right)}{\left|\Phi^{*}(x, s)-\Phi^{*}(y, s)\right|^{2}}\right| \\
& \leq c_{0}\left|(\Phi(x, s)-\Phi(y, s))-\left(\Phi^{*}(x, s)-\Phi^{*}(y, s)\right)\right||x-y|^{-3} \\
& \leq c_{0}\|\rho(s)\|_{1, \Omega_{0}}|x-y|^{-2} .
\end{aligned}
$$

It follows that the quantity

$$
\begin{aligned}
& \int_{\Omega_{s}} \frac{\sigma^{\perp}(\Phi(x, s)-y)}{|\Phi(x, s)-y|^{2}}(\omega(y, s)-\omega(\Phi(x, s), s)) d y \\
& -\int_{\Omega_{s}^{*}} \frac{\sigma^{\perp}\left(\Phi^{*}(x, s)-y\right)}{\left|\Phi^{*}(x, s)-y\right|^{2}}\left(\omega^{*}(y, s)-\omega^{*}\left(\Phi^{*}(x, s), s\right)\right) d y
\end{aligned}
$$

is bounded by, since $\omega_{0} \in C^{\alpha}$,

$$
\begin{aligned}
& c_{0}\|\rho(s)\|_{1, \Omega_{0}} \int_{\Omega_{0}} \frac{\left|\omega_{0}(y)-\omega_{0}(x)\right|}{|x-y|^{2}} d y \\
& \leq c_{0}\|\rho(s)\|_{1, \Omega_{0}} \int_{\Omega_{0}} \frac{1}{|x-y|^{2-\alpha}} d y=c_{0}\|\rho(s)\|_{1, \Omega_{0}} .
\end{aligned}
$$

Hence, we obtain from (3.23) that

$$
\begin{aligned}
& \left|\nabla u_{0}(\Phi(x, s), s)-\nabla u_{0}^{*}\left(\Phi^{*}(x, s), s\right)\right| \\
& \leq c_{0}\|\rho(s)\|_{1, \Omega_{0}}+\left|k_{1}(x, s)\right|+\left|k_{2}(x, s)\right|,
\end{aligned}
$$

where

$$
\begin{aligned}
k_{1}(x, s) & =\hat{b} \int_{\Omega_{s} \backslash \Phi\left(B_{\varepsilon}(x), s\right)} \frac{\sigma^{\perp}(\Phi(x, s)-y)}{|\Phi(x, s)-y|^{2}} d y \\
& -\hat{b} \int_{\Omega_{s}^{*} \backslash \Phi^{*}\left(B_{\varepsilon}(x), s\right)} \frac{\sigma^{\perp}\left(\Phi^{*}(x, s)-y\right)}{\left|\Phi^{*}(x, s)-y\right|^{2}} d y \\
k_{2}(x, s) & =\hat{b} \int_{\Phi\left(\Omega_{0} \cap B_{\varepsilon}(x), s\right)} \frac{\sigma^{\perp}(\Phi(x, s)-y)}{|\Phi(x, s)-y|^{2}} d y \\
& -\hat{b} \int_{\Phi^{*}\left(\Omega_{0} \cap B_{\varepsilon}(x), s\right)} \frac{\sigma^{\perp}\left(\Phi^{*}(x, s)-y\right)}{\left|\Phi^{*}(x, s)-y\right|^{2}} d y
\end{aligned}
$$


and $\varepsilon$ is a positive constant to be determined later. By changes of variable, we have

$$
\begin{aligned}
k_{1}(x, s) & =\hat{b} \int_{\Omega_{0} \backslash B_{\varepsilon}(x)} \frac{\sigma^{\perp}(\Phi(x, s)-\Phi(y, s))}{|\Phi(x, s)-\Phi(y, s)|^{2}} J(\Phi(y, s)) d y \\
& -\hat{b} \int_{\Omega_{0} \backslash B_{\varepsilon}(x)} \frac{\sigma^{\perp}\left(\Phi^{*}(x, s)-\Phi^{*}(y, s)\right)}{\left|\Phi^{*}(x, s)-\Phi^{*}(y, s)\right|^{2}} J\left(\Phi^{*}(y, s)\right) d y .
\end{aligned}
$$

Hence, by (3.25),

$$
\begin{aligned}
\left|k_{1}(x, s)\right| & \leq c_{0}\|\rho(s)\|_{1, \Omega_{0}} \int_{\varepsilon}^{d\left(\Omega_{0}\right)} \frac{1}{r} d r \\
& \leq c_{0}\|\rho(s)\|_{1, \Omega_{0}}(1+|\log \varepsilon|) .
\end{aligned}
$$

To estimate $k_{2}(x, t)$, we consider first the case that $x \in \Omega_{0}$ and

$$
\operatorname{dist}\left(x, \partial \Omega_{0}\right) \geq \varepsilon \text {. }
$$

For this fixed $x, B_{\varepsilon}(x) \subset \Omega_{0}$. Since

$$
\int_{B_{r}(0)} \frac{\sigma^{\perp}(y)}{|y|^{2}} d y=0, \quad r>0,
$$

we have

$$
\begin{aligned}
k_{2}(x, s)= & \hat{b} \int_{\Phi\left(B_{\varepsilon}(x), s\right)} \frac{\sigma^{\perp}(\Phi(x, s)-y)}{|\Phi(x, s)-y|^{2}} d y-\hat{b} \int_{\Phi^{*}\left(B_{\varepsilon}(x), s\right)} \frac{\sigma^{\perp}\left(\Phi^{*}(x, s)-y\right)}{\left|\Phi^{*}(x, s)-y\right|^{2}} d y \\
= & \hat{b} \int_{\left(\Phi\left(B_{\varepsilon}(x), s\right)-\Phi(x, s)\right) \backslash B_{d(x)}(0)} \frac{\sigma^{\perp}(y)}{|y|^{2}} d y \\
- & \hat{b} \\
& \int_{\left(\Phi^{*}\left(B_{\varepsilon}(x), s\right)-\Phi^{*}(x, s)\right) \backslash B_{d(x)}(0)} \frac{\sigma^{\perp}(y)}{|y|^{2}} d y \\
= & \hat{b} \int_{\Sigma_{1}} \frac{\sigma^{\perp}(y)}{|y|^{2}} d y-\hat{b} \int_{\Sigma_{2}} \frac{\sigma^{\perp}(y)}{|y|^{2}} d y,
\end{aligned}
$$

where

$$
d(x)=\min \left(\operatorname{dist}\left(\Phi(x, s), \partial \Phi\left(B_{\varepsilon}(x), s\right)\right), \operatorname{dist}\left(\Phi^{*}(x, s), \partial \Phi^{*}\left(B_{\varepsilon}(x), s\right)\right)\right),
$$

and

$$
\begin{aligned}
& \Sigma_{1}=\left[\left(\Phi\left(B_{\varepsilon}(x), s\right)-\Phi(x, s)\right) \backslash B_{d(x)}(0)\right] \backslash\left[\left(\Phi^{*}\left(B_{\varepsilon}(x), s\right)-\Phi^{*}(x, s)\right)\right], \\
& \Sigma_{2}=\left[\left(\Phi^{*}\left(B_{\varepsilon}(x), s\right)-\Phi^{*}(x, s)\right) \backslash B_{d(x)}(0)\right] \backslash\left[\left(\Phi\left(B_{\varepsilon}(x), s\right)-\Phi(x, s)\right)\right] .
\end{aligned}
$$

Since $\Phi \in B(M, T)$, it is easy to see that there exist two positive constants $c_{1}$ and $c_{2}$ depending only on $M, T$ such that

$$
c_{1} \varepsilon \leq d(x) \leq c_{2} \varepsilon .
$$


For any unit vector $\nu \in R^{2}$, set

$$
\Lambda_{i}(\nu)=\sup \left\{r: r \nu \in \Sigma_{i}\right\}, \lambda_{i}(\nu)=\inf \left\{r: r \nu \in \Sigma_{i}\right\}, i=1,2 .
$$

By (3.29), $\lambda_{i}(\nu) \geq d(x) \geq c_{1} \varepsilon$. Let $x_{1} \in \partial B_{\varepsilon}(x)$ such that

$$
\Lambda_{1}(\nu) \nu=\Phi\left(x_{1}, s\right)-\Phi(x, s)=\nabla \Phi\left(y_{1}\right)\left(x_{1}-x\right),
$$

where $y_{1}$ is a point lying in the line segment connecting $x$ and $x_{1}$. Without loss of generality, we may assume that there exists $x_{1}^{*} \in \partial B_{\varepsilon}(x)$ such that

$$
\lambda_{1}(\nu) \nu=\Phi^{*}\left(x_{1}^{*}, s\right)-\Phi^{*}(x, s)=\nabla \Phi^{*}\left(y_{1}^{*}\right)\left(x_{1}^{*}-x\right),
$$

where $y_{1}^{*}$ is a point lying in the line segment connecting $x$ and $x_{1}^{*}$. Hence,

$$
\begin{aligned}
\Lambda_{1}(\nu)-\lambda_{1}(\nu) & =\left|\nabla \Phi\left(y_{1}\right)^{-1} \nu\right|^{-1}\left|x_{1}-x\right|-\left|\nabla \Phi^{*}\left(y_{1}^{*}\right)^{-1} \nu\right|^{-1}\left|x_{1}^{*}-x\right| \\
& =\varepsilon\left(\left|\nabla \Phi\left(y_{1}\right)^{-1} \nu\right|^{-1}-\left|\nabla \Phi^{*}\left(y_{1}^{*}\right)^{-1} \nu\right|^{-1}\right) \\
& \leq c_{0} \varepsilon\left|\nabla \Phi\left(y_{1}\right)^{-1} \nu-\nabla \Phi^{*}\left(y_{1}^{*}\right)^{-1} \nu\right| \\
& \leq c_{0} \varepsilon\left|\nabla \Phi\left(y_{1}\right)-\nabla \Phi^{*}\left(y_{1}^{*}\right)\right| \\
& \leq c_{0} \varepsilon\left|y_{1}-y_{1}^{*}\right|^{\alpha} \leq c_{0} \varepsilon^{1+\alpha}
\end{aligned}
$$

It follows that

$$
\begin{aligned}
\left|\int_{\Sigma_{1}} \frac{\sigma^{\perp}(y)}{|y|^{2}} d y\right| & \leq\left|\int_{\partial B_{1}(0)} \int_{\lambda_{1}(\nu)}^{\Lambda_{1}(\nu)} \frac{1}{r^{2}} r d r d \nu\right| \leq\left|\int_{\partial B_{1}(0)} \log \left(\frac{\Lambda_{1}(\nu)}{\lambda_{1}(\nu)}\right) d \nu\right| \\
& \leq c_{0}\left|\int_{\partial B_{1}(0)} \frac{\Lambda_{1}(\nu)-\lambda_{1}(\nu)}{\lambda_{1}(\nu)} d \nu\right| \leq c_{0} \varepsilon^{\alpha}
\end{aligned}
$$

Analogously, we have

$$
\left|\int_{\Sigma_{2}} \frac{\sigma^{\perp}(y)}{|y|^{2}} d y\right| \leq c_{0} \varepsilon^{\alpha} .
$$

Inserting this into (3.28), we obtain

$$
\left|k_{2}(x, s)\right| \leq c_{0} \varepsilon^{\alpha} .
$$

Substituting this and (3.27) into (3.26), we obtain, for dist $\left(x, \partial \Omega_{0}\right) \geq \varepsilon$,

$$
\left|\nabla u_{0}(\Phi(x, s), s)-\nabla u_{0}^{*}\left(\Phi^{*}(x, s), s\right)\right| \leq c_{0}\|\rho(s)\|_{1, \Omega_{0}}(1+|\log \varepsilon|)+c_{0} \varepsilon^{\alpha} .
$$

Since $\nabla u_{0}$ and $\nabla u_{0}^{*}$ belong to $C^{\alpha}$, the above estimate remains true for all $x \in \Omega_{0}$. We now choose $\varepsilon^{\alpha}=\|\rho(s)\|_{1, \Omega_{0}}$. The above inequality, together with (3.24) and (3.15), leads to

$$
\left|\nabla u(\Phi(x, s), s)-\nabla u^{*}\left(\Phi^{*}(x, s), s\right)\right| \leq c_{0}\|\rho(s)\|_{1, \Omega_{0}}\left(1+\left|\log \|\rho(s)\|_{1, \Omega_{0}}\right|\right) .
$$


Hence, by (3.20),

$$
\begin{aligned}
& \left|\nabla F(\Phi)(x, t)-\nabla F\left(\Phi^{*}\right)(x, t)\right| \\
& \leq c_{0} \int_{0}^{t}\|\rho(s)\|_{1, \Omega_{0}}\left(1+\left|\log \|\rho(s)\|_{1, \Omega_{0}}\right|\right) d s .
\end{aligned}
$$

Step 4: $F$ has a unique fixed point.

We can now define a sequence $\left\{\Phi_{n}\right\}$ by

$$
\Phi_{1}(x, t)=x, \Phi_{n+1}(x, t)=F\left(\Phi_{n}\right)(x, t) .
$$

Set

By (3.22) and (3.30), we have

$$
\rho_{n}(t)=\left\|\left(\Phi_{n+1}-\Phi_{n}\right)(t)\right\|_{1, \Omega_{0}}
$$

$$
\begin{aligned}
\rho_{n+1}(t) & =\left\|\left(\nabla F\left(\Phi_{n+1}\right)-\nabla F\left(\Phi_{n}\right)\right)(t)\right\|_{1, \Omega_{0}} \\
& \leq c_{0} \int_{0}^{t} \rho_{n}(s) \log \left(2+\rho_{n}(s)\right) d s .
\end{aligned}
$$

By [6, Sec. 9], this implies that there exists a positive number $T^{*} \leq T$ such that the sequence is convergent in $C_{x}^{1}\left(\Omega_{0}\right)$ norm, for $t \leq T^{*}$. By (3.22), this limit is a fixed point of the mapping $F$. The above inequality also implies that the fixed point is unique. This fixed point is clearly the solution of (2.7). The proof is complete.

REMARK 3.1. For the integral system studied in [5], it was shown that the sequence $\left\{\hat{\rho}_{n}(t)\right\}_{n=1}$ defined by

$$
\hat{\rho}_{n}(t)=\left\|\left(\Phi_{n+1}-\Phi_{n}\right)(t)\right\|_{0, \Omega_{0}}
$$

satisfies inequality (3.31). Hence Step 3, i.e., the estimate of $\nabla F(\Phi)-\nabla F\left(\Phi^{*}\right)$ is not needed in proving local existence. For the 2D Euler system, the authors of [1] actually assumed local existence based on a result in [3].

From the proof of Theorem 3.1, we can see that the existence time $T$ depends only on the Hölder norms of the initial data. Hence, à priòri bounds on $\|\Phi(t)\|_{1+\alpha, \Omega_{0}}$ and $\left\|\Phi^{-1}(t)\right\|_{1, \Omega_{t}}$ for $t<T$ will guarantee that the solution can be extended beyond $T$. In fact, we have

Corollary 3.1. Let $\Phi(x, t)$ be the $C^{1+\alpha}$ solution of (2.7) for $t<T$. If

$$
\theta=\sup _{t<T}\|\Phi(t)\|_{1+\alpha, \Omega_{0}}+\sup _{t<T}\left\|\Phi^{-1}(t)\right\|_{1, \Omega_{t}}
$$

is finite, then the solution $\Phi(x, t)$ may be extended for $t<T+T_{0}$ for some positive constants $T_{0}$ depending only on $\theta$.

Proof. Let $T^{*}<T$ be a constant, and let $\omega^{*}(x)=\omega_{0}\left(\Phi^{-1}\left(x, T^{*}\right)\right)$, where $\Phi(x, t)$ is the $C^{1+\alpha}$ solution of (2.7) for $t<T$. Consider the mapping

$$
\begin{aligned}
& F^{*}\left(\Phi^{*}\right)(x, t)=\Phi\left(x, T^{*}\right) \\
& +\int_{0}^{t} \int_{\Omega_{s}^{*}} \frac{1}{b\left(\Phi^{*}(x, s)\right)} \nabla^{\perp} K\left(\Phi^{*}(x, s), z\right) b(z)^{2} \omega^{*}\left(\Phi^{*-1}(z, s)\right) d z d s,
\end{aligned}
$$


defined in the set

$$
\begin{array}{r}
B^{*}\left(M, T_{1}\right)=\left\{\Phi^{*}(x, t) \in R^{2}:\left\|\Phi^{*}(t)\right\|_{1+\alpha, \Omega_{0}},\left\|\Phi^{*}(x, \cdot)\right\|_{\alpha} \leq M,\right. \\
\left.\Phi^{*}(x, 0)=\Phi\left(x, T^{*}\right),\left|\nabla \Phi^{*}(x, t)-\nabla \Phi\left(x, T^{*}\right)\right| \leq \frac{1}{2 \theta}\right\} .
\end{array}
$$

For any $\Phi^{*} \in B^{*}(M, T)$, we have

$$
\begin{aligned}
& \left|\nabla \Phi^{*}(x, t)\left(\nabla \Phi\left(x, T^{*}\right)\right)^{-1}-I_{2}\right| \\
& \leq\left|\nabla \Phi^{-1}\left(\Phi\left(x, T^{*}\right), T^{*}\right)\right|\left|\nabla \Phi^{*}(x, t)-\nabla \Phi\left(x, T^{*}\right)\right| \leq 1 / 2 .
\end{aligned}
$$

Therefore, $\Phi^{*}(\cdot, t)$ is invertible and $\left\|\Phi^{*-1}(t)\right\|_{1, \Omega_{t}} \leq c_{0} M^{2}$, where $c_{0}$ is a constant independent of $T$ and $M$. Using the same method as in the proof of Theorem 3.1, one may show that there exists an $M$ and $T_{1}$ depending on $\theta$ (but not on the particular choice of $\left.T^{*}\right)$ such that $F^{*}$ maps $B^{*}(M, T)$ into itself and that $F^{*}$ admits a unique fixed point $\Phi^{*}(x, t)$. Now we select $T_{0}=3 T_{1} / 4$ and $T^{*}=\max \left(T-T_{1} / 4,0\right)$. The mapping $\Phi^{*}(x, t)$ is then the extension of $\Phi(x, t)$.

4. Global Existence of the $C^{1+\alpha}$ Solution. In this section, we shall show that the $C^{1+\alpha}$ solution obtained in the previous section can be extended to all $t>0$.

Lemma 4.1. For any $T>0$, there exist positive constants $\eta(T)$ and $\beta(T)$ with $\beta(T) \leq \alpha$, depending only on $T$ and the initial data $\delta_{\Omega_{0}, \alpha},\left\|\omega_{0}\right\|_{\alpha, \Omega_{0}}$ and $b(x)$ such that for any $C^{1+\alpha}$ solution $\Phi(x, t)$ of $(2.7)$ for $t<T_{0}$, we have

$$
d\left(\Omega_{t}\right)+\|\omega(t)\|_{\beta(T), \Omega_{t}} \leq \eta(T) \quad\left(\text { for } t<\min \left(T_{0}, T\right)\right),
$$

where $\omega(x, t)=\omega_{0}\left(\Phi^{-1}(x, t)\right)$ and $d\left(\Omega_{t}\right)$ is the diameter of the region $\Omega_{t}$.

Proof. Since $\omega(x, t)=\omega_{0}\left(\Phi^{-1}(x, t)\right)$, it follows $|\omega(x, t)| \leq\left\|\omega_{0}\right\|_{0, \Omega_{0}}$. By $(2.7)$ and (3.15), we write

$$
\Phi(x, t)=x+\int_{0}^{t} u_{0}(\Phi(x, s), t) d s+\int_{0}^{t} u_{1}(\Phi(x, s), t) d s,
$$

where $u_{0}$ and $u_{1}$ have the expressions (3.16) and (3.17). It is easy to see that

$$
\left|u_{0}(x, t)\right| \leq c_{0} \int_{\Omega_{t}}\left|\nabla_{x}^{\perp} \log \right| x-y|| d y \leq c_{0} \int_{0}^{d\left(\Omega_{t}\right)} d s=c_{0} d\left(\Omega_{t}\right)
$$

for a constant $c_{0}$ dependent of the initial data only. By (3.2), we have

$$
\left|u_{1}(x, t)\right| \leq c_{0} \int_{\Omega_{t}}\left|\nabla_{x}^{\perp} K_{1}(x, y)\right| d y \leq c_{0}\left(d\left(\Omega_{t}\right)+1\right) .
$$

Hence, for $x \in \Omega_{0}$,

$$
|\Phi(x, t)| \leq|x|+\int_{0}^{t}|u(\Phi(x, s), t)| d s \leq c_{0}+c_{0} \int_{0}^{t}\left(d\left(\Omega_{t}\right)+1\right) d s .
$$


It follows that

$$
d\left(\Omega_{t}\right)=\sup _{x, y \in \Omega_{0}}|\Phi(x, t)-\Phi(y, t)| \leq c_{0}+c_{0} \int_{0}^{t}\left(d\left(\Omega_{t}\right)+1\right) d s .
$$

The Gronwall lemma guarantees that $d\left(\Omega_{t}\right) \leq \eta(T)$, where $\eta(T)=c_{0} e^{c_{0} T}$ depends only on $T$ and the initial data. In what follows, $\eta(T)$ may vary. But it depends only on $T$ and initial data.

By the well-known results in [15] (see also [13]), $u_{0}$ is quasi-Lipschitz, i.e.,

$$
\left|u_{0}(x, t)-u_{0}(y, t)\right| \leq c_{0}|x-y||\log | x-y||,
$$

for $|x-y| \leq 1 / 2$. By (3.2), we have, for $|x-y| \leq 1 / 2$,

$$
\begin{aligned}
\left|u_{1}(x, t)-u_{1}(y, t)\right| & \leq\left\|\omega_{0}\right\|_{0, \Omega_{0}}\left|b^{-1}(x)-b^{-1}(y)\right| \int_{\Omega_{t}}\left|\nabla_{x}^{\perp} K_{1}(x, z) d z\right| \\
& +\left\|\omega_{0}\right\|_{0, \Omega_{0}}\left|b^{-1}(y)\right| \int_{\Omega_{t}}\left|\nabla_{x}^{\perp} K_{1}(x, z)-\nabla_{x}^{\perp} K_{1}(y, z) d z\right| \\
& \leq \eta(T)|x-y| .
\end{aligned}
$$

It follows that

$$
\begin{gathered}
\left|\frac{d|\Phi(x, t)-\Phi(y, t)|}{d t}\right| \leq|u(\Phi(x, t), t)-u(\Phi(y, t), t)| \\
\leq \eta(T)|\Phi(x, t)-\Phi(y, t)||\log | \Phi(x, t)-\Phi(y, t)||,
\end{gathered}
$$

for $|\Phi(x, t)-\Phi(y, t)| \leq 1 / 2$. By the Gronwall lemma, we deduce that

$$
\eta(T)|x-y|^{1 / \mu(t)} \leq|\Phi(x, t)-\Phi(y, t)| \leq \eta(T)|x-y|^{\mu(t)},
$$

for $|x-y|^{\mu(t)} \leq(2 \eta(T))^{-1}$, where $\mu(t)=\exp (-\eta(T) t)$. Since $\Phi^{-1}(x, t) \in \Omega_{0}$ is bounded, this inequality implies that $\left\|\Phi^{-1}(t)\right\|_{\mu(t), \Omega_{t}} \leq \eta(T)$. Combining this with the fact that $\Phi(x, t)$ is bounded (due to (4.1)), since $\mu(T) \leq \mu(t)$ for $t<T$, we obtain $\left\|\Phi^{-1}(t)\right\|_{\mu(T), \Omega_{t}} \leq \eta(T)$. We now choose

$$
\beta(T)=\alpha \mu(T) .
$$

Since $\omega(x, t)=\omega_{0}\left(\Phi^{-1}(x, t)\right)$, it follows that

$$
\|\omega(t)\|_{\beta(T), \Omega_{t}} \leq c_{0}\left\|\omega_{0}\right\|_{\alpha, \Omega_{0}}\left\|\Phi^{-1}(t)\right\|_{\mu(T), \Omega_{t}}^{\alpha} \leq \eta(T) .
$$

The assertion follows from this inequality and (4.1).

Lemma 4.2. Let $\Phi(x, t)$ be the $C^{1+\alpha}$ solution (2.7) for $t<T$, and $\omega(x, t)=\omega_{0}\left(\Phi^{-1}(x, t)\right)$. Suppose that there exist constants $\eta>0$ and $0<\beta \leq \alpha$ such that for $t<T$,

$$
d\left(\Omega_{t}\right)+\|\omega(t)\|_{\beta, \Omega_{t}} \leq \eta<\infty .
$$

Then

$$
\left\|\Phi^{-1}(t)\right\|_{1, \Omega_{t}}+\delta_{\Omega_{t}, \beta} \leq c(\eta, \beta, T),
$$


where $\delta_{\Omega_{t}, \beta}$ is defined as $\delta_{\Omega_{t}}$ in (3.3) in which the $\alpha$-Hölder seminorm $|\cdot|_{\alpha}$ is replaced by $\beta$-Hölder seminorm $|\cdot|_{\beta}$, and $c(\eta, \beta, T)$ is a constant depending only on $\eta, \beta, T$, and the initial data.

Proof. Let $\Omega_{0}=\left\{\varphi_{0}<0\right\}$ for some $C^{1+\alpha}$ function $\varphi_{0}$ such that $\nabla \varphi_{0} \neq 0$ on $\partial \Omega_{0}$. We recall that $\Omega_{t}=\{\varphi(\cdot, t)<0\}$ where $\varphi(x, t)=\varphi_{0}\left(\Phi^{-1}(x, t)\right)$. Let $u=u_{0}+u_{1}$ be the velocity as in (3.15)-(3.17). By (3.23), we have

$$
\begin{aligned}
\nabla u_{0}(x, t) \nabla^{\perp} \varphi(x, t) & =\hat{b} \nabla \int_{\Omega_{t}} \nabla^{\perp} \log |x-y| \omega(y, t) d y \nabla^{\perp} \varphi(x, t) \\
& =\hat{b} \int_{\Omega_{t}} \frac{\sigma^{\perp}(x-y)}{|x-y|^{2}}(\omega(y, t)-\omega(x, t)) d y \nabla^{\perp} \varphi(x, t) \\
& +\pi \hat{b} \omega(x, t) \nabla \varphi(x, t)+\hat{b} \int_{\Omega_{t}} \frac{\sigma^{\perp}(x-y)}{|x-y|^{2}} d y \nabla^{\perp} \varphi(x, t) \omega(x, t) \\
& =k_{1}+k_{2}+k_{3} .
\end{aligned}
$$

Note that $\nabla^{\perp} \varphi(x, t)$ is weakly divergence free in $\Omega_{t}$ and tangent to $\partial \Omega_{t}$. We obtain from $[1$, Corollary 1] that

$$
k_{3}=\hat{b} \int_{\Omega_{t}} \frac{\sigma^{\perp}(x-y)}{|x-y|^{2}}\left(\nabla^{\perp} \varphi(x, t)-\nabla^{\perp} \varphi(y, t)\right) d y \omega(x, t),
$$

and consequently that, by Lemma 3.1 and (4.2),

$$
\begin{aligned}
\left|k_{3}\right|_{\beta} \leq c_{0}\left(|\omega(t)|_{\beta}|\nabla \varphi(t)|_{0}+|\omega(t)|_{0}|\nabla \varphi(t)|_{\beta}\right) \\
\cdot \log \left(2+\beta^{-1}|\nabla \varphi(t)|_{\beta} d\left(\Omega_{t}\right)+d\left(\Omega_{t}\right) \delta_{\Omega_{t}, \beta}\right) \\
\leq c(\eta, \beta)\|\nabla \varphi(t)\|_{\beta} \log \left(2+|\nabla \varphi(t)|_{\beta}+\delta_{\Omega_{t}, \beta}\right),
\end{aligned}
$$

where $c(\eta, \beta)$ depends only on $\eta, \beta$, and the initial data. In particular, $c(\eta, \beta)$ is bounded by $c(\eta) \log \beta^{-1}$, for a constant $c(\eta)$ depending only on $\eta$ and the initial data. In the above inequality, as well as in what follows, we may omit the subscript: $\|\cdot\|_{\beta}=\|\cdot\|_{\beta, \Omega_{t}}$. Analogously, we have

$$
\begin{aligned}
\left|k_{1}\right|_{\beta} & \leq c_{0}\left(|\omega(t)|_{\beta}|\nabla \varphi(t)|_{0}+|\omega(t)|_{0}|\nabla \varphi(t)|_{\beta}\right) \\
& \cdot \log \left(2+\beta^{-1}|\omega(t)|_{\beta} d\left(\Omega_{t}\right)+d\left(\Omega_{t}\right) \delta_{\Omega_{t}, \beta}\right) \\
& \leq c(\eta, \beta)\|\nabla \varphi(t)\|_{\beta} \log \left(2+\delta_{\Omega_{t}, \beta}\right) .
\end{aligned}
$$

It follows that

$$
\left|\nabla u_{0} \nabla^{\perp} \varphi(t)\right|_{\beta} \leq c(\eta, \beta)\|\nabla \varphi(t)\|_{\beta} \log \left(2+\|\nabla \varphi(t)\|_{\beta}+\delta_{\Omega_{t}, \beta}\right) .
$$

By (3.2), it is easy to see that

$$
\left|\nabla u_{1}(t)\right|_{\beta} \leq c(\eta, \beta)
$$


Hence

$$
\left|\nabla u_{1} \nabla^{\perp} \varphi(t)\right|_{\beta} \leq c(\eta, \beta)\|\nabla \varphi(t)\|_{\beta},
$$

and consequently, this and (4.4) lead to

$$
\left|\nabla u \nabla^{\perp} \varphi(t)\right|_{\beta} \leq c(\eta, \beta)\|\nabla \varphi(t)\|_{\beta} \log \left(2+\|\nabla \varphi(t)\|_{\beta}+\delta_{\Omega_{t}, \beta}\right) .
$$

The same argument also shows that $\left|\nabla u \nabla^{\perp} \varphi(t)\right|_{0}$ is bounded by the right-hand side of the above inequality. Hence,

$$
\left\|\nabla u \nabla^{\perp} \varphi(t)\right\|_{\beta} \leq c(\eta, \beta)\|\nabla \varphi(t)\|_{\beta} \log \left(2+\|\nabla \varphi(t)\|_{\beta}+\delta_{\Omega_{t}, \beta}\right) .
$$

Next, by differentiating $\varphi(\Phi(x, t), t)=\varphi_{0}(x)$ with respect to $t$, we obtain

$$
\frac{\partial \varphi(x, t)}{\partial t}+u(x, t) \cdot \nabla \varphi(x, t)=0 .
$$

Hence $\nabla \varphi$ solves

$$
\frac{\partial \nabla \varphi}{\partial t}+(u \cdot \nabla) \nabla \varphi=-(\nabla u)^{\top} \nabla \varphi
$$

where $(\nabla u)^{\top}$ is the transpose matrix of $\nabla u$. It follows that

$$
\nabla \varphi(\Phi(x, t), t)=\nabla \varphi_{0}(x)-\int_{0}^{t}\left((\nabla u)^{\top} \nabla \varphi\right)(\Phi(x, s), s) d s
$$

or equivalently,

$$
\nabla \varphi(x, t)=\nabla \varphi_{0}\left(\Phi^{-1}(x, t)\right)-\int_{0}^{t}\left((\nabla u)^{\top} \nabla \varphi\right)\left(\Phi\left(\Phi^{-1}(x, t), s\right), s\right) d s .
$$

Note that (4.6) holds only in the distribution sense since $\varphi \in C^{1+\alpha}$. By using test functions as we did in the proof of Proposition 2.1, one may verify that (4.7) holds. Since

$$
\frac{\partial \nabla \Phi(x, t)}{\partial t}=\nabla u(\Phi(x, t), t) \nabla \Phi
$$

it follows that, for $0<s<t$,

$$
\begin{aligned}
\exp \left(-\int_{s}^{t}|\nabla u(\tau)|_{0} d \tau\right) & \leq\left|\nabla\left(\Phi\left(\Phi^{-1}(x, t), s\right)\right)\right| \\
& \leq \exp \left(\int_{s}^{t}|\nabla u(\tau)|_{0} d \tau\right)
\end{aligned}
$$


Therefore

$$
\begin{aligned}
|\nabla \varphi(t)|_{\beta} & \leq\left|\nabla \varphi_{0}\right|_{\beta}\left|\nabla \Phi^{-1}(t)\right|_{0}^{\beta} \\
& +\int_{0}^{t}\left|(\nabla u)^{\top} \nabla \varphi(s)\right|_{\beta}\left|\nabla\left(\Phi\left(\Phi^{-1}(\cdot, t), s\right)\right)\right|_{0}^{\beta} d s \\
& \leq\left|\nabla^{\perp} \varphi_{0}\right|_{\beta} \exp \left(\beta \int_{0}^{t}|\nabla u(\tau)|_{0} d \tau\right) \\
& +\int_{0}^{t}\left|(\nabla u)^{\top} \nabla \varphi(s)\right|_{\beta} \exp \left(\beta \int_{s}^{t}|\nabla u(\tau)|_{0} d \tau\right) d s .
\end{aligned}
$$

It is obvious that, by (4.7),

$$
|\nabla \varphi(t)|_{0} \leq\left|\nabla \varphi_{0}\right|_{0}+\int_{0}^{t}\left|(\nabla u)^{\top} \nabla \varphi(s)\right|_{0} d s .
$$

We have arrived at

$$
\begin{aligned}
\|\nabla \varphi\|_{\beta} & \leq\left\|\nabla \varphi_{0}\right\|_{\beta} \exp \left(\beta \int_{0}^{t}|\nabla u(\tau)|_{0} d \tau\right) \\
& +\int_{0}^{t}\left\|(\nabla u)^{\top} \nabla \varphi(s)\right\|_{\beta} \exp \left(\beta \int_{s}^{t}|\nabla u(\tau)|_{0} d \tau\right) d s .
\end{aligned}
$$

Introducing the notation $(a, b)^{\perp}=(b, a)$, a direct computation leads to

$$
\left((\nabla u)^{\top} \nabla \varphi\right)^{\perp}=(\nabla \cdot u) \nabla^{\perp} \varphi-\nabla u \nabla^{\perp} \varphi
$$

Since $\nabla \cdot(b u)=0$, we have

$$
\nabla \cdot u=b^{-1} \nabla \cdot(b u)+b u \cdot \nabla b^{-1}=b u \cdot \nabla b^{-1} .
$$

Hence,

$$
\left((\nabla u)^{\top} \nabla \varphi\right)^{\perp}=\left(b u \cdot \nabla b^{-1}\right) \nabla^{\perp} \varphi-\nabla u \nabla^{\perp} \varphi .
$$

It follows from Lemma 3.2 that

$$
\begin{aligned}
\left\|\left(b u \cdot \nabla b^{-1}\right) \nabla^{\perp} \varphi(t)\right\|_{\beta} & \leq c(\eta, \beta)\left(\|u(t)\|_{\beta}\|\nabla \varphi(t)\|_{\beta}\right) \\
& \leq c(\eta, \beta)\|\nabla \varphi(t)\|_{\beta} \log \left(2+\delta_{\Omega_{t}, \beta}\right) .
\end{aligned}
$$

Combining this with (4.5) and (4.10), we obtain

$$
\left\|(\nabla u)^{\top} \nabla \varphi(t)\right\|_{\beta} \leq c(\eta, \beta)\|\nabla \varphi(t)\|_{\beta} \log \left(2+\|\nabla \varphi(t)\|_{\beta}+\delta_{\Omega_{t}, \beta}\right) .
$$


Substituting this into (4.9), it follows that

$$
\begin{aligned}
\|\nabla \varphi(t)\|_{\beta} & \leq\left\|\nabla \varphi_{0}\right\|_{\beta} \exp \left(\beta \int_{0}^{t}|\nabla u(\tau)|_{0} d \tau\right) \\
& +c(\eta, \beta) \int_{0}^{t}\|\nabla \varphi(s)\|_{\beta, \Omega_{s}} \log \left(2+\|\nabla \varphi(s)\|_{\beta, \Omega_{s}}+\delta_{\Omega_{s}, \beta}\right) \\
& \cdot \exp \left(\beta \int_{s}^{t}|\nabla u(\tau)|_{0} d \tau\right) d s .
\end{aligned}
$$

Multiplying by $\exp \left(-\beta \int_{0}^{t}|\nabla u(\tau)|_{0} d \tau\right)$, the above inequality leads to

$$
\begin{gathered}
\|\nabla \varphi(t)\|_{\beta} \exp \left(-\beta \int_{0}^{t}|\nabla u(\tau)|_{0} d \tau\right) \leq\left\|\nabla \varphi_{0}\right\|_{\beta} \\
+c(\eta, \beta) \int_{0}^{t} \exp \left(-\beta \int_{0}^{s}|\nabla u(\tau)|_{0} d \tau\right)\|\nabla \varphi(s)\|_{\beta, \Omega_{s}} \\
\cdot \log \left(2+\|\nabla \varphi(s)\|_{\beta, \Omega_{s}}+\delta_{\Omega_{s}, \beta}\right) d s .
\end{gathered}
$$

Recall that, by definition (3.3) and (4.8),

$$
\begin{aligned}
\delta_{\Omega_{t}, \beta} & =\frac{|\nabla \varphi(t)|_{\beta}}{|\nabla \varphi(t)|_{\text {inf, }, \partial \Omega_{t}}} \leq \frac{|\nabla \varphi(t)|_{\beta}}{\left|\nabla \Phi^{-1} \nabla \varphi_{0}\left(\Phi^{-1}(t)\right)\right|_{\text {inf }, \partial \Omega_{t}}} \\
& \leq c_{0} \exp \left(\int_{0}^{t}|\nabla u(\tau)|_{0} d \tau\right)|\nabla \varphi(t)|_{\beta} .
\end{aligned}
$$

Introduce the functions

$$
\begin{aligned}
& h(t)=\beta \int_{0}^{t}|\nabla u(\tau)|_{0} d \tau, \\
& f(t)=\|\nabla \varphi(t)\|_{\beta, \Omega_{t}} \exp (-h(t))+1 .
\end{aligned}
$$


From (4.11) and (4.12), we thus arrive at the following inequality:

$$
\begin{aligned}
f(t) & \leq c_{0}+c(\eta, \beta) \int_{0}^{t} f(s) \log \left(2+\|\nabla \varphi(s)\|_{\beta, \Omega_{s}}+\delta_{s, \beta}\right) d s \\
& \leq c_{0}+c(\eta, \beta) \int_{0}^{t} f(s) \log \left(2+f(s) \exp \left(\left(1+\beta^{-1}\right) h(s)\right)\right) d s \\
& \leq c_{0}+c(\eta, \beta) \int_{0}^{t} f(s)(1+h(s)+\log f(s)) d s .
\end{aligned}
$$

Denote by $g(t)$ the function on the right-hand side of the above inequality:

$$
g(t)=c_{0}+c(\eta, \beta) \int_{0}^{t} f(s)(1+h(s)+\log f(s)) d s .
$$

Then

$$
\begin{aligned}
g^{\prime}(t) & =c(\eta, \beta) f(t)(1+h(t)+\log f(t)) \\
& \leq c(\eta, \beta) g(t)(1+h(t)+\log g(t))
\end{aligned}
$$

It follows that

$$
\frac{d}{d t} \log g(t) \leq c(\eta, \beta)(1+h(t))+c(\eta, \beta) \log g(t) .
$$

Integrating this inequality, we obtain

$$
\log g(t) \leq c(\eta, \beta) e^{c(\eta, \beta) t}\left(1+\int_{0}^{t}(1+h(s)) e^{-c(\eta, \beta) s} d s\right) .
$$

Since $f \leq g$ and $b(t)$ is increasing, it follows that, for $t<T$,

$$
\begin{aligned}
\log f(t) & \leq c(\eta, \beta, T)\left(1+h(t) \int_{0}^{t} e^{-c(\eta) s} d s\right) \\
& \leq c(\eta, \beta, T)(1+h(t)),
\end{aligned}
$$

where $c(\eta, \beta, T)$ depends only on $c(\eta, \beta)$ and $T$. Using this inequality and recalling the definitions of $h(t)$ and $f(t)$ and applying Lemma (3.2) and (4.12), we arrive at

$$
\begin{aligned}
h(t) & =\beta \int_{0}^{t}|\nabla u(s)|_{0, \Omega_{s}} d s \leq c(\eta, \beta) \int_{0}^{t} \log \left(2+\delta_{\Omega_{s}, \beta}\right) d s \\
& \leq c(\eta, \beta) \int_{0}^{t}(\log f(s)+h(s)) d s \leq c(\eta, \beta, T) \int_{0}^{t}(1+h(s)) d s .
\end{aligned}
$$

The standard Gronwall's inequality implies

$$
h(t) \leq c(\eta, \beta, T) .
$$


Therefore, by (4.13), $f(t) \leq c(\eta, \beta, T)$. Consequently, by the definitions of $f(t)$ and $h(t)$, and by (4.12), we find that $\delta_{\Omega_{t}, \beta}$ is bounded by $c(\eta, \beta, T)$, a constant depending on $\eta, \beta$ and $T$. Taking $s=0$ in (4.8), it follows that $\left|\Phi^{-1}(t)\right|_{1 . \Omega_{t}} \leq \exp \left(\beta^{-1} h(t)\right) \leq c(\eta, \beta, T)$. The proof is complete.

REMARK 4.1. For $2 \mathrm{D}$ constant vortex patches, since $\omega(x, t)$ is constant in $\bar{\Omega}_{t}$, Lemma 4.1 holds automatically, and Lemma 4.2 can be established directly for $\beta=\alpha$ using the incompressibility condition $\nabla \cdot u=0$ (sec [3]).

Theorem 4.1. Then there exists a unique global $C^{1+\alpha}$ solution $\Phi(x, t)$ of $(2.7)$ for all $t>0$. Consequently, there exists a unique global weak solution $(\omega, u)$ for the lake equation. Furthermore, the solution has the regularity $\omega(\cdot, t) \in C^{\alpha}\left(\bar{\Omega}_{t}\right) . u(\cdot, t) \in$ $C^{1+\alpha}\left(\bar{\Omega}_{t}\right), \partial \Omega_{t} \in C^{1+\alpha}$ for all $t>0$.

Proof. It suffices to show that for any $T>0$, there exists a unique $C^{1+\alpha}$ solution $\Phi(x, t)$ of $(2.7)$ for all $t<T$. By Theorem 3.1, we know that there exists a unique $C^{1+\alpha}$ solution $\Phi(x, t)$ of $(2.7)$ for $t<T_{0}$ for some $T_{0}>0$. We assume $T_{0}<T$, since otherwise the proof is complete. By Lemma 4.1 and 4.2 , there exist $0<\beta(T) \leq \alpha$ and $c(T)>0$, depending only on the initial data and $T$ such that

$$
d\left(\Omega_{t}\right)+\|\omega(t)\|_{\beta(T), \Omega_{t}}+\left\|\Phi^{-1}(t)\right\|_{1, \Omega_{t}}+\delta_{\Omega_{t}, \beta(T)} \leq c(T) .
$$

Let $u$ be the velocity defined in (3.15). By Lemma 3.2 with $\beta(T)$ replacing $\alpha$, the above estimate implies $|\nabla u(t)|_{0, \Omega_{t}} \leq c(T)$. It follows that

$$
\begin{aligned}
|\nabla \Phi(t)|_{0, \Omega_{0}} & \leq c_{0}+\int_{0}^{t}|\nabla u(s)|_{0 . \Omega \Omega_{s}}|\nabla \Phi(s)|_{0 . \Omega_{0}} d s \\
& \leq c_{0}+c(T) \int_{0}^{t}|\nabla \Phi(s)|_{0 . \Omega_{0}} d s .
\end{aligned}
$$

Hence, $|\nabla \Phi(t)|_{0 . \Omega_{0}} \leq c(T)$ for $t<T_{0}<T$. Using this and Lemma 3.2 again, we obtain

$$
\begin{aligned}
|\nabla \Phi(t)|_{\alpha, \Omega_{0}} \leq & c_{0}+\int_{0}^{t}|\nabla u(\Phi(\cdot, s), s)|_{0, \Omega_{0}}|\nabla \Phi(s)|_{\alpha . \Omega_{0}} d s \\
& +\int_{0}^{t}|\nabla u(\Phi(\cdot, s), s)|_{\alpha, \Omega_{0}}|\nabla \Phi(s)|_{0, \Omega_{0}} d s \\
& \leq c_{0}+c(T) \int_{0}^{t}\left(|\nabla \Phi(s)|_{\alpha, \Omega_{0}}+|\nabla u(s)|_{\alpha . \Omega_{s}}\right) d s \\
& \leq c_{0}+c(T) \int_{0}^{t}\left(\delta_{\Omega_{s} . \alpha}+\|\omega(s)\|_{\alpha, \Omega_{s}}+|\nabla \Phi(s)|_{\alpha, \Omega_{0}}\right) \\
& \cdot \log \left(2+\|\omega(s)\|_{\alpha . \Omega_{s}}+\delta_{\Omega_{s} . \alpha}\right) d s .
\end{aligned}
$$


Note that $\omega(x, t)=\omega_{0}\left(\Phi^{-1}(x, t)\right)$ and, by definition $(3.3)$,

$$
\delta_{\Omega_{t}, \alpha}=\frac{|\nabla \varphi(t)|_{\alpha, \Omega_{t}}}{|\nabla \varphi(t)|_{\text {inf }, \partial \Omega_{t}}}, \varphi(x, t)=\varphi_{0}\left(\Phi^{-1}(x, t)\right) .
$$

By (4.8), we have

$$
\begin{aligned}
|\omega(t)|_{\alpha, \Omega_{t}} & \leq\left|\omega_{0}\right|_{\alpha, \Omega_{0}}\left|\nabla \Phi^{-1}(t)\right|_{1, \Omega_{t}}^{\alpha} \leq c(T), \\
\delta_{\Omega_{t}, \alpha} & \leq|\nabla \varphi(t)|_{\alpha, \Omega_{t}} \exp \int_{0}^{t}|\nabla u(s)|_{0, \Omega_{s}} d s \leq c(T)|\nabla \Phi(t)|_{\alpha, \Omega_{0}} .
\end{aligned}
$$

Therefore,

$$
|\nabla \Phi(t)|_{\alpha, \Omega_{0}} \leq c_{0}+c(T) \int_{0}^{t}\left(1+|\nabla \Phi(s)|_{\alpha, \Omega_{0}}\right) \log \left(2+|\nabla \Phi(s)|_{\alpha, \Omega_{0}}\right) d s .
$$

This inequality implies that $|\nabla \Phi(t)|_{\alpha, \Omega_{0}} \leq c(T)$. It then follows from Corollary 3.1 that there exists a $T_{1}>0$, depending only on $c(T)$ and the initial data such that the solution $\Phi(x, t)$ can be extended, still in the class of $C^{1+\alpha}$, to $t<T_{0}+T_{1}$. We emphasize again that $c(T)$ depends only on $T$ and the initial data. Since $T_{1}$ is independent of $T_{0}$, we may repeat this procedure to extend the solution to $t<T_{0}+n T_{1}$, until $T_{0}+n T_{1}$ exceeds the given $T$, where $n$ is a positive integer. The proof is complete.

\section{REFERENCES}

[1] A. L. Bertozzi and P. Constantin, Global regularity for vortex patches, Comm. Math. Phys. 152 (1993), 19-28.

[2] R. Camassa, D. D. Holm, and C. D. Levermore, Long-time shallow water equations with a varying bottom, J. Fluid Mech. 349 (1997), 173-189

[3] J.-Y. Chemin, Sur le mouvement des particules d'un fluide parfait incompressible bidimensionnel, Invention Math. 103 (1991), 599-629.

[4] E. A. Coddington and J. L. Levinson, Theory of Ordinary Differential Equations, McGraw-Hill, New York, 1995.

[5] A. Friedman and C. Huang, Averaged motion of charged particles under their self-induced electric field, Indiana Univ. Math. J. 43 (1994), 1167-1225.

[6] A. Friedman and J. L. Velázquez, A time-dependent free boundary problem modeling the visual image in electrophotography, Archive Rat. Meth. Anal. 123 (1993), 259-303.

[7] C. Huang, On boundary regularity of non-constant vortex patches, Comm. Appl. Math. 3 (1999), 449-459.

[8] C. Huang and T. Svobodny, Evolution of mixed-state region in type-II superconductors, SIAM J. Math. Anal. 29 (1998), 1002-1021.

[9] S. Itô, Diffusion Equations, Translations of Mathematical Monographs 114, Providence, RI: Amer. Math. Soc., 1992.

[10] C. D. Levermore and M. Oliver, Analyticity of solutions for a generalized Euler equation, J. Differential Equations 133 (1997), 321-339.

[11] C. D. Levermore, M. Oliver, and E. S. Titi, Global well-posedness for the lake equations, Phys. D 98 (1996), 492-502.

[12] C. D. Levermore, M. Oliver and E. S. Titi, Global well-posedness for models of shallow water equations, Indiana Univ. Math. J., Vol. 45 (1996), 479-510.

[13] A. Majda, Vorticity and the mathematical theory of incompressible fluid flow, Comm. Pure Appl. Math. 39 (1986), S187-220.

[14] M. Oliver, Classical solutions for a generalized Euler equation in two dimensions, J. Math. Anal. Appl. 215 (1997), 471-483. 
[15] V. I. Yudovich, Non-stationary flow of an ideal incompressible liquid, Zh. Vych. Mat. 3 (1963), 1032-1066 (In Russian). 